\title{
Enzyme Histochemistry on Normal and Pathological Human Thymic Tissues
}

\author{
Rita Rezzani, Luigi Rodella, Cristina Agostini and Rossella Bianchi \\ Department of Biomedical Sciences and Biotechnology, University of Brescia, Brescia, Italy
}

Received for publication September 26, 1996 and in revised form July 30, 1997

\begin{abstract}
The human thymuses, the well differentiated carcinoma, the thymoma with spindle epithelial cells and the undifferentiated thymoma have been examined by histochemical techniques [lactate dehydrogenase (LDH), succinate dehydrogenase (SDH), acid phosphatase (AcP), alkaline phosphatase (ALP) and dihydrofolate dehydrogenase (DHFR)] to study the metabolic changes due to the pathological conditions in the epithelial cells (EPCs) and accessory cells. In all three different types of studied thymomas the EPCs showed a more intense LDH and SDH positivity than in normal thymus. These data indicate a possible damage of respiratory chain energy-coupling mechanism. The EPCs presented a different pattern (negative or very strong) of ALP and AcP

positivity both in normal and pathological thymuses. These results suggest that the EPCs are a heterogenous population presenting a variety of functional activities both in normal and pathological conditions. The macrophages showed a different pattern (very weak, weak and moderate) in dehydrogenase and in hydrolytic enzymes both in normal and tumour cells. The macrophages were uniformly distributed throughout the thymoma but it is interesting to note that in the well differentiated thymomas these cells were localized in high number only around the neoplastic nodules that were proliferating. These findings suggest that the macrophages are important for defending the organism against the formation of new neoplastic nodules.
\end{abstract}

Key words: Thymomas, Human thymus, Histochemistry, Epithelial cells, Macrophages

\section{Introduction}

The thymus provides the microenvironment in which immature, bone marrrow derived, thymocyte precursor cells proliferate and differentiate into mature immuno-competent T-lymphocytes, which then leave the thymus and find their place in the immune system $[3,15]$. The importance of thymus non lymphoid cells for thymocyte maturation has been studied for a long time. The thymic accessory cells (epithelial cells [11] macrophages [1] and interdigitating cells $[16,17])$ appear to influence at least part of the maturation process, perhaps through their expression of class II histocompatibility antigens.

Thymomas are epithelial tumours of the thymus which contain variable proportions of lymphocytes. The architectural complexity of thymomas reflects the ability of neoplastic epithelial cells (EPCs) to interact with lymphoid cells in an attempt to organize a thymus-like structure [13]. This suggestion has received further support

Correspondence to: Prof. Rossella Bianchi, Division of Human Anatomy, Department of Biomedical Sciences and Biotechnology, Via Valsabbina, 19, 25123 Brescia Italy. from the immunohistochemical demonstration that neoplastic epithelial cells and thymoma-associated lymphocytes share morphological and antigenic properties with their counterparts of the normal thymus $[10,18]$. All these findings suggest that thymus lymphocyte maturation may also take place in thymomas, and that the accessory cells could participate in this process [16]. The aim of the present study was to better understand the metabolic activities both of the accessory cells and of the lymphocytes in the human thymoma using conventional histochemical methods. Moreover, a comparative analysis of the pathological results with the normal controls was carried out. The possible difference in metabolic activity could indicate a particular stage of maturation or differentiation of the EPCs and also be a marker for the degree of malignancy of the tumour.

\section{Materials and Methods}

\section{Patients}

Fresh samples of thymomas were obtained from 12 patients of both sexes ( 8 men and 4 women aged between 35 and 56) undergoing surgery because of an anterior 
mediastinal mass; in each case, multiple fragments of the tumours were snap-frozen in liquid nitrogen. Their histological classification (well differentiated carcinomas, thymomas with spindle epithelial cells, undifferentiated thymomas) was made according to $\mathrm{HO}$ et al. [9]. Three well differentiated carcinomas, 5 thymomas with spindle epithelial cells and 4 undifferentiated thymomas were recognized.

Fresh samples of normal thymus were obtained from 7 cardiac surgery patients: 3 young patients (with ages ranging between 7 and 12) and 4 adults patients (with age ranging between 27 and 40 ). The histology of the thymus was normal and the patients did not suffer from any immunological disorder.

\section{Histochemical and histological study}

All samples were cut in a cryostat to a thickness of $10 \mu \mathrm{m}$. The seriate sections were analyzed with haematoxylin-eosin and histochemically with acid phosphatase (AcP), alkaline phospatase (ALP), lactate dehydrogenase (LDH), succinate dehydrogenase (SDH) and dihydrofolate reductase (DHFR) activity.

\section{Acid phosphatase}

AcP (orthophosphoric monoester phosphohydrolase, EC 3.1.3.2) [6] catalyzes the hydrolysis of esters of orthophosphoric acid with various alcohol and phenols [7]. To demonstrate AcP activity, tissue sections were fixed in $60 \%$ acetone for $2 \mathrm{~min}$ followed by incubation in $2.2 \mathrm{mM}$ naphtol AS-BI phosphate as substrate and $3.8 \mathrm{mM}$ Fast Red Violet as capture agent dissolved in $0.1 \mathrm{M}$ acetate buffer pH 5.0.

\section{Alkaline phosphatase}

ALP (orthophosphoric monoester phosphohydrolase, EC 3.1.3.1) catalyzes the breakdown of orthophosphoric acid esters [6] in cell membrane transport processes. To demonstrate ALP activity, tissue sections were fixed in $60 \%$ acetone for $2 \mathrm{~min}$ followed by incubation in $2.2 \mathrm{mM}$ naphtol AS-BI phosphate as substrate and $3.8 \mathrm{mM}$ Fast Red Violet as capture agent dissolved in $0.2 \mathrm{M}$ Tris buffer, pH 10.0.

Control reactions (without the addition of substrate) were performed for each enzyme assay.

\section{Lactate dehydrogenase}

LDH (L-lactate: NAD oxidoreductase, EC 1.1.127) [2] catalyzes the interconversion of pyruvate and lactate during glycolysis [4]. To demonstrate LHD activity unfixed tissue sections were incubated with the following medium: $1 \mathrm{mM}$ nitro blue tetrazolium (NBT), $1 \mathrm{mM}$ sodium lactate, $4.5 \mathrm{mM}$ magnesium chloride, $1.3 \mathrm{mM}$ nicotinamide adenine dinucleotide (NAD) dissolved in phosphate buffer $0.05 \mathrm{M} \mathrm{pH}$ 7.4. The incubation was carried out at $37^{\circ} \mathrm{C}$ for $30 \mathrm{~min}$.

\section{Succinate dehydrogenase}

SDH, (succinate: oxidoreductase, EC 1.399.1) [2] catalyzes the conversion of succinate into fumarate in the citric acid cycle. To demonstrate SDH activity unfixed tissue sections were incubated in the same medium as for LDH but with a change of substrate $(1 \mathrm{mM}$ sodium succinate) and no coenzyme. The incubation was carried out at $37^{\circ} \mathrm{C}$ for $30 \mathrm{~min}$.

\section{Dihydrofolate reductase}

DHFR (tetrahydrofolate dehydrogenase; 5,6,7,8tetrahydrofolate: NADP oxidoreductase, EC 1.51.3) [5] plays an important role in purine and pyrimidine metabolism and is present particularly in proliferating and differentiating cells. To demonstrate DHFR activity, tissue sections, fixed in $60 \%$ acetone for $30 \mathrm{~s}$, were incubated with the following medium: $1 \mathrm{mM}$ tetrahydrofolic acid (dissolved in $0.1 \%$ mercaptoethanol prior to use); $1 \mathrm{mM}$ NADP, M/15 phosphate buffer with a final $\mathrm{pH} 7.4$, $0.3 \mathrm{mM}$ NBT, $0.2 \mathrm{mM}$ phenazine methosulphate (PMS), $5 \mathrm{mM}$ magnesium chloride and $10 \mathrm{mM}$ sodium azide. The incubation was carried out in a nitrogen atmosphere for $15 \mathrm{~min}$ at $37^{\circ} \mathrm{C}$.

In the frozen sections we identified the different cell populations (IDCs, macrophages and EPCs) on the basis of their morphological structure, using haematoxylineosin staining and their immunophenotype, using two monoclonal antibodies recognising the dendritic cells and the macrophages (anti human dendritic cells -CD35) and the epithelial cells (anti human cytokeratin).

\section{Results}

\section{Normal thymuses}

The morphology of the thymus was clearly recognisable only in young human studied; a light medulla and a dark cortex were found. The cortical lymphocytes showed the features of lymphoblasts with a large amount of cytoplasm and a round nucleus, while the medullary lymphocytes had only a rim of cytoplasm and a round nucleus. A large quantity of macrophages (Mo) was present in the cortex and a small quantity and in the medulla. The EPCs were observed in the cortex, in the medulla. Their morphological structure was similar. The interdigitating cells (IDCs) were present only in the medulla. Thymic tissues from adult human showed islands of lympho-epithelial tissue with cortex and medulla not well differentiated scattered in fatty tissue (Fig. 1).

\section{Acid phosphatase}

A marked staining reaction was seen in macrophages both in the cortex and in the medulla (Fig. 2). The IDCs were only very slightly positive. The cortical and medullary thymocytes were clearly positive to AcP. Their positivity was characteristically demonstrated as a dot in one pole of the cytoplasm. The positivity of the EPCs of 
the cortex was very high. A weak positive reaction was also observed in the thymic EPCs constituting Hassall's corpuscles.
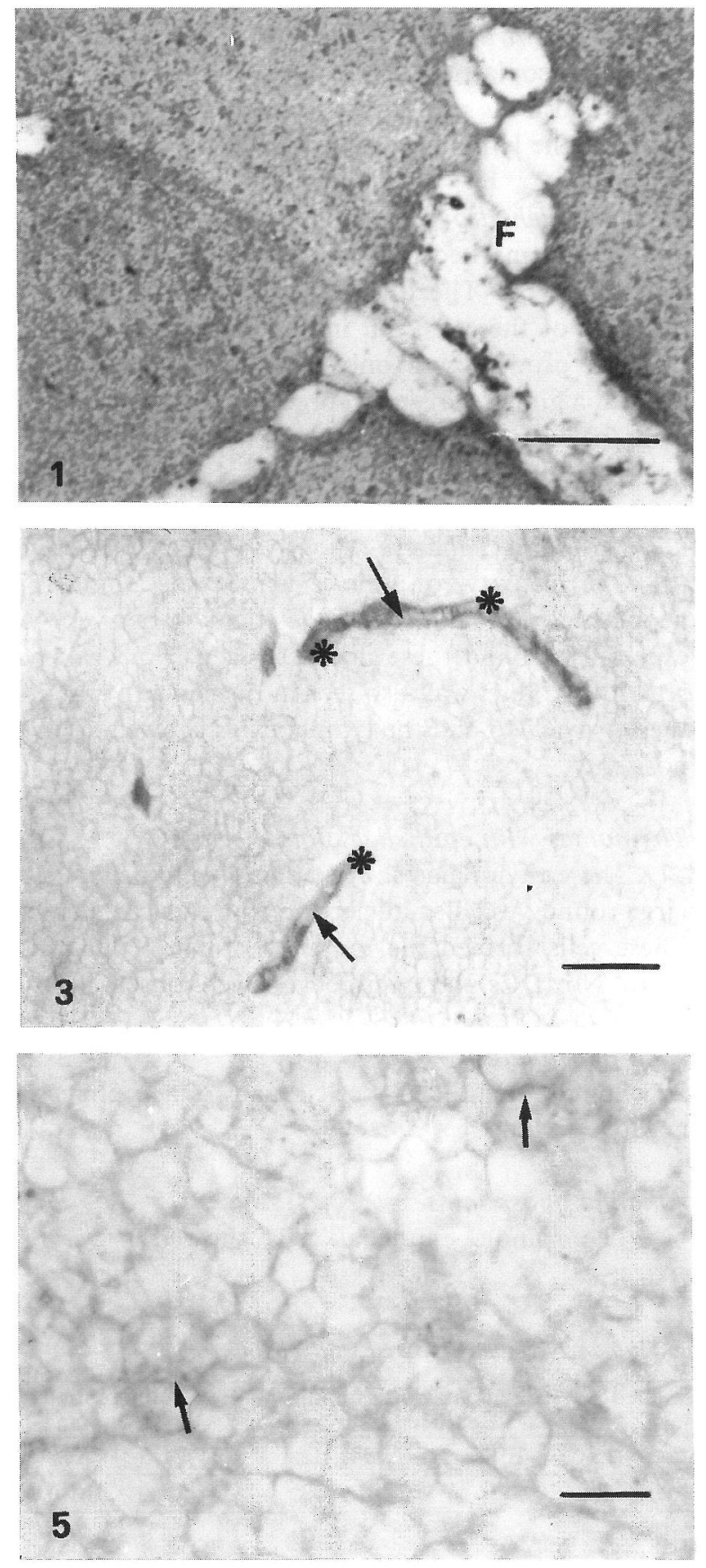

Alkaline phosphatase

Weak positivity was occasionally observed in the EPCs making up the Hassall's corpuscles and in the cells lining the vessels (Fig. 3). No ALP activity was detected in the lymphocytes or in the accessory cells.
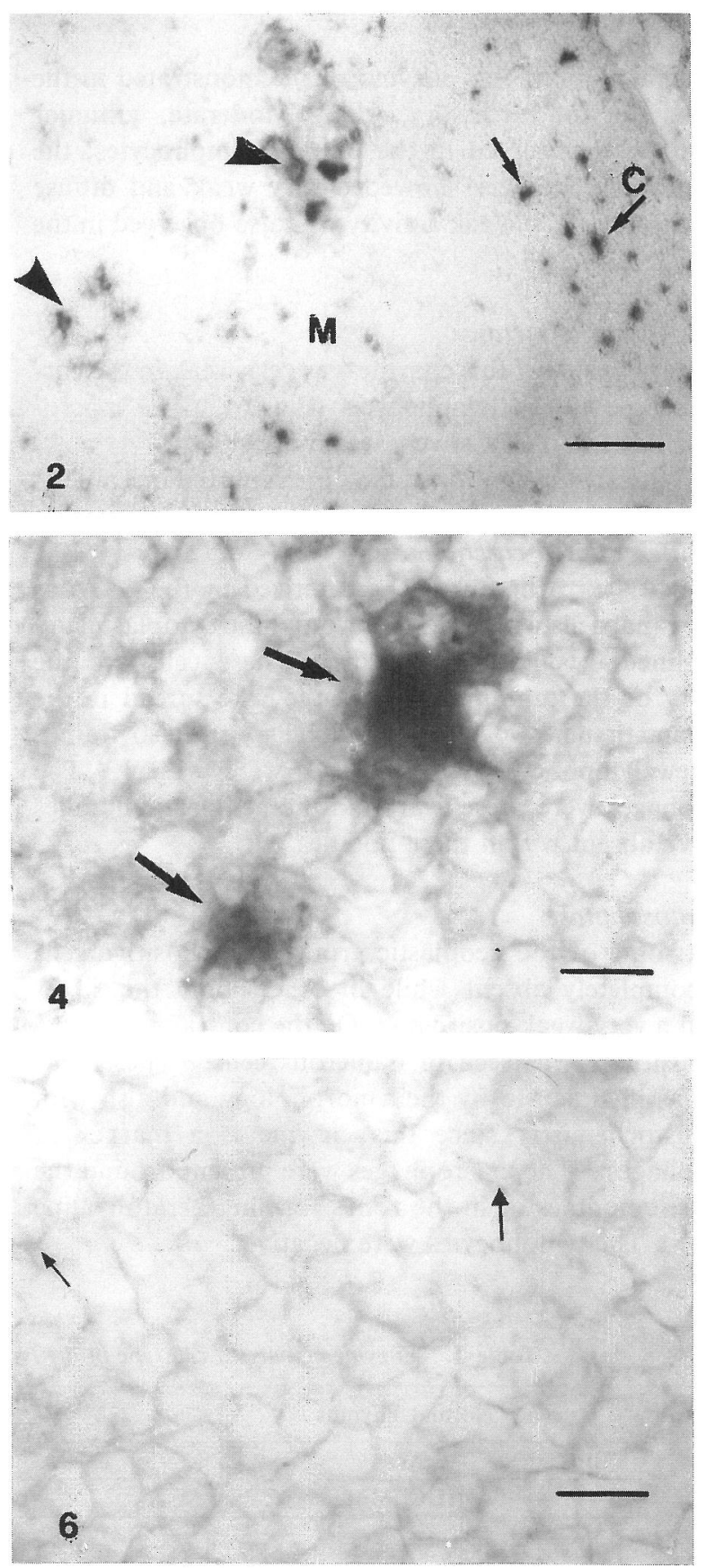

Fig. 1. Photomicrograph of lympho-epithelial tissue of normal adult thymus showing cortex and medulla not well differentiated. $F=$ fatty tissue infiltration. Haematoxylin eosin staining (bar: $100 \mu \mathrm{m}$ ).

Fig. 2. Photomicrograph showing AcP positive cells (macrophages: arrows; IDCs: arrowheads) in cortex (C) and medulla (M) of young human thymus (bar: $50 \mu \mathrm{m}$ ).

Fig. 3. Photomicrograph showing ALP positive cells $\left(^{*}\right)$ around the vessels (arrows) in the medulla of young human thymus (bar: $\left.50 \mu \mathrm{m}\right)$.

Fig. 4. Photomicrograph showing LDH positive cells (IDCs arrows) in the medulla of young human thymus (bar: $12.5 \mu \mathrm{m}$ ).

Fig. 5. Photomicrograph showing SDH positive cells in the medulla of young human thymus (arrows) (bar: $12.5 \mu \mathrm{m}$ ).

Fig. 6. Photomicrograph showing a very weak DHFR positivity in the cortex of young human thymus (arrows) (bar: $12.5 \mu \mathrm{m}$ ). 


\section{Lactate dehydrogenase}

The activity of this enzyme was demonstrated in the cytoplasm of the thymocytes. The lymphocytes in the cortex showed a very weak positivity similar to that observed in the medulla. The thymic EPCs were weakly positive, while the IDCs and Mo showed strong activity (Fig. 4).

\section{Succinate dehydrogenase}

The activity of this enzyme was demonstrated in the lymphocytes and accessory cells. Moderate, granular positivity was observed in the cortical lymphocytes; the medullary lymphocytes showed a very weak and diffuse positivity (Fig. 5). Weak activity was also observed in the EPCs, IDCs and Mo.

\section{Dihydrofolate reductase}

The intensity of this enzyme was very weak in the cortical and medullary lymphocytes (Fig. 6). The macrophages, IDCs and EPCs were weakly positive.

All the above mentioned data are reported in Table 1.

\section{Well differentiated carcinomas}

This type of tumour is characterized by the presence of polygonal cells with clear or eosinophilic cytoplasm and well-defined cytoplasmic margins. These cells are the majority of the tumour cells and they are spread in the malignant tissue. Spindle shaped cells were also found. Very few lymphocytes and no IDCs were present. The macrophages were present around the neoplastic nodules but were absent within these nodules.

\section{Acid phosphatase}

In some of the neoplastic groups AcP positive cells were completely absent while in others only the EPCs showed a very weak positivity. On the contrary, a strong AcP positivity was seen in numerous cells. These cells were classified as Mo by their morphology and also with AcP histochemistry since this enzyme is a marker of macrophages. The macrophages were present around the neoplastic nodules or in the zones forming keratohyaline (Fig. 7). The lymphocytes were negative.

\section{Alkaline phosphatase}

The neoplastic cells were negative to this enzyme; a strong and diffuse positivity was seen in the accessory cells. The granular positivity was observed in the Mo and polygonal cells of the zones forming keratohyaline. The thymocytes showed a very weak positivity.

\section{Lactate dehydrogenase}

The neoplastic cells showed a very strong positivity (Fig. 8), also the macrophages were very positive. On the contrary the lymphocytes were weakly positive.

\section{Succinate dehydrogenase}

A very marked positivity was seen in the neoplastic cells (Fig. 9). The diffuse positivity was found in the majority of the cells, but in some neoplastic nodules the reaction product was only granular. The spindle cells showed a granular reaction product similar to that observed in the macrophages. The lymphocytes had a very weak positivity.

\section{Dihydrofolate reductase}

The activity of this enzyme was found in the neoplastic nodules. The positivity was very strong especially around these nodules, even if a weak positive reaction product was also seen at the centre of the some of these. The Mo and the lymphocytes showed a very weak positivity.

\section{Thymomas with epithelial spindle cells}

These thymomas showed spindle epithelial cells with large round vesicular nuclei and thin nuclear membranes. These cells formed the majority of the cellular population. No IDCs and lymphocytes were observed.

\section{Acid phosphatase}

Spindle epithelial cells and macrophages were negative to AcP.

\section{Alkaline phosphatase}

The spindle epithelial cells showed a very strong

Table 1. Enzyme activity of cell type in the Normal Human Thymus compared with Thymomas

\begin{tabular}{|c|c|c|c|c|c|c|c|c|c|c|c|c|c|}
\hline \multirow{3}{*}{ Enzyme } & \multicolumn{4}{|c|}{ Normal Thymus } & \multicolumn{3}{|c|}{$\begin{array}{l}\text { Well differentiated } \\
\text { Carcinoma }\end{array}$} & \multicolumn{3}{|c|}{$\begin{array}{l}\text { Thymomas with spindle } \\
\text { Epithelial Cells }\end{array}$} & \multicolumn{3}{|c|}{$\begin{array}{c}\text { Undifferentiated } \\
\text { Thymoma }\end{array}$} \\
\hline & \multicolumn{2}{|c|}{ EPCs } & \multirow{2}{*}{$\begin{array}{c}\text { Acs } \\
\text { (IDC, Mo) }\end{array}$} & Ls & EPCs & Acs & Ls & EPCs & Acs & Ls & EPCs & Acs & Ls \\
\hline & $\mathrm{C}$ & $\mathrm{M}$ & & C $\quad \mathrm{M}$ & & (Mo) & & & (Mo) & & & (Mo) & \\
\hline $\mathrm{AcP}$ & +++ & $+/-$ & $+1+1+1$ & $+/-+/-$ & $+/-$ & +++ & 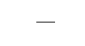 & - & - & N.F. & +++ & +++ & + \\
\hline ALP & $+/-$ & $+/-$ & - & $-\quad-$ & - & +++ & $+/-$ & +++ & - & N.F. & $+/-$ & +++ & - \\
\hline LDH & + & + & +++ & ++ & +++ & +++ & + & +++ & +++ & N.F. & +++ & +++ & $+/-$ \\
\hline SDH & + & + & + & $+\quad+/-$ & +++ & +++ & $+/-$ & +++ & ++ & N.F. & +++ & ++ & $+/-$ \\
\hline DHFR & + & + & + & $+/-+/-$ & +1 & $+/-$ & $+/-$ & $+\backslash+++$ & $+/-$ & N.F. & $+\backslash+++$ & ++ & - \\
\hline
\end{tabular}

Intensity of AcP, ALP, LDH, SDH and DHFR reaction in normal thymus and thymomas; N.F. $=$ not found; $+<-$ very weak; + weak; ++ moderate; +++ strong; $C=$ Cortex, $M=$ Medulla, Acs=accessory cells (IDCs=interdigitating cells, Mo=macrophages), Ls=lymphocytes, $\mathrm{EPCs}=$ epithelial cells 

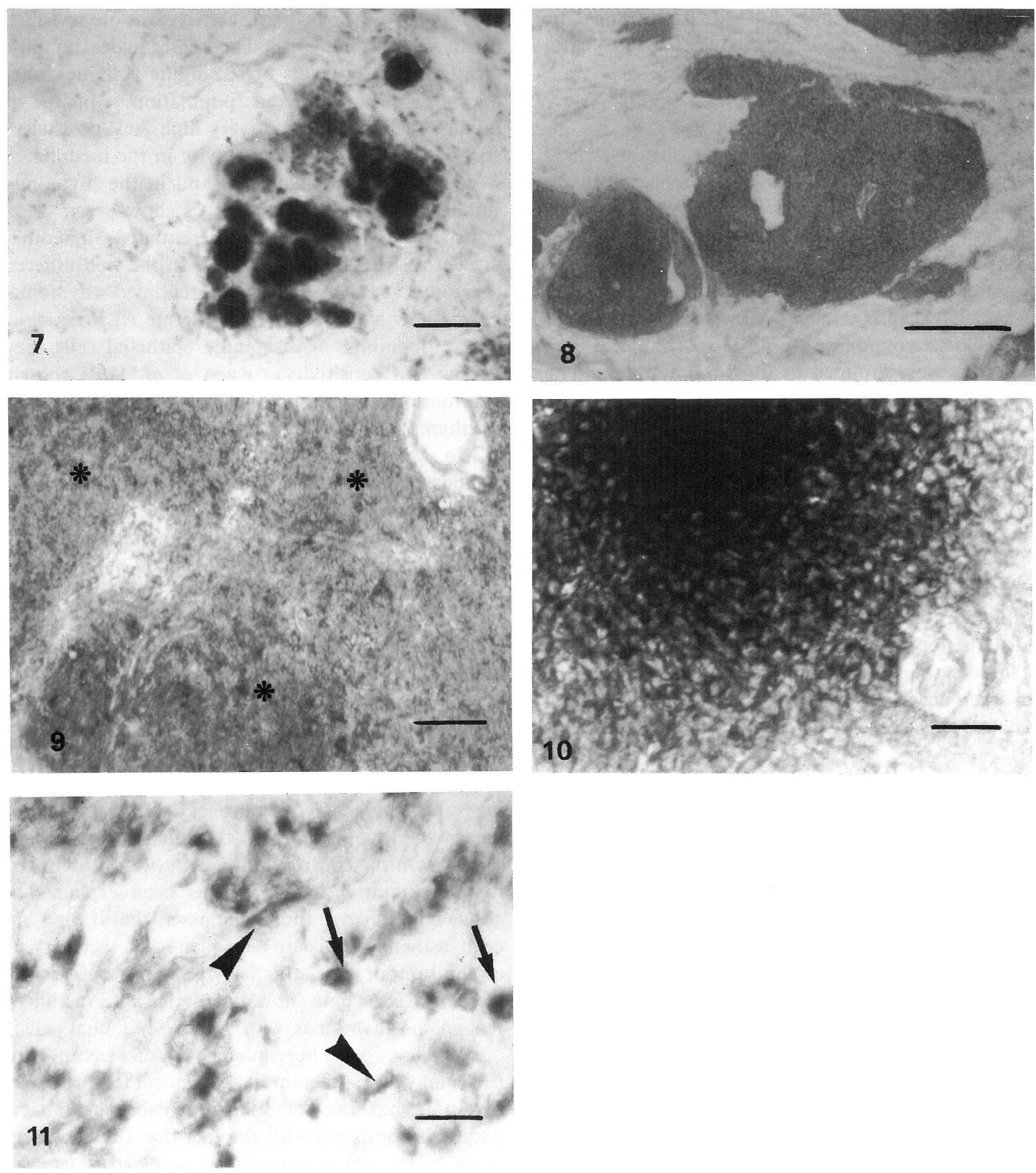

Fig. 7. Photomicrograph showing AcP positive macrophages, in the well differentiated carcinoma (bar: $12.5 \mu \mathrm{m}$ ).

Fig. 8. Photomicrograph showing the LDH positive neoplastic areas of the well differentiated carcinoma (bar: $100 \mu \mathrm{m}$ ).

Fig. 9. Photomicrograph showing the SDH positivity in the neoplastic areas $(*)$ of the well differentiated carcinoma (bar: $50 \mu \mathrm{m})$.

Fig. 10. Photomicrograph showing DHFR positivity in a neoplastic nodule of thymoma with epithelial spindle cells (bar: $25 \mu \mathrm{m}$ ).

Fig. 11. Photomicrograph showing AcP positive cells (macrophages=arrows; EPCs=arrowheads) in undifferentiated thymoma (bar: $12.5 \mu \mathrm{m}$ ).

positivity also near the vessels. The reaction product appeared either as diffuse cellular staining or as coarse cytoplasmic granules of formazan. The Mo were negative and they were spread in the pathological tissue.

\section{Lactate dehydrogenase}

The neoplastic cells presented a strong positivity.
The reaction product showed a diffuse staining. The Mo were also positive, but they were clearly recognizable because of their morphology.

\section{Succinate dehydrogenase}

The spindle neoplastic cells showed a strong positivity. Their positivity was evident as a diffuse staining or 
sometimes as the cytoplasmic granules of formazan. The Mo, which were spread in the pathological tissue, had a moderate positivity.

\section{Dihydrofolate reductase}

The neoplastic cells were weakly positive and there were only some groups of cells showing a strong positivity (Fig. 10). The Mo showed a very weak positivity.

\section{Undifferentiated thymomas}

These thymomas showed neoplastic cells with nuclei of different shape surrounded by abundant cytoplasm. These cells had round or spindle morphology and sometimes they were grouped to form the nodules. The pathological tissue presented a large number of cells that had the features of macrophages.

\section{Acid phosphatase}

The Mo had a very strong positivity; the epithelial cells and the thymocytes were also very positive (Fig. 11).

\section{Alkaline phosphatase}

A different behaviour of the neoplastic cells was observed. The round cells had a weak positivity while the cells in spindle shape were negative to ALP. The Mo and the other accessory cells were positive. The lymphocytes were negative.

\section{Lactate dehydrogenase}

Both the round and spindle cells showed a strong positivity; the macrophages were very positive and localized in the centre of the neoplastic nodules. The lymphocytes showed a very weak positivity.

\section{Succinate dehydrogenase}

The accessory cells had a moderate granular positivity while the round and spindle cells showed a strong granular positivity. The lymphocytes showed a very weak positivity.

\section{Dihydrofolate reductase}

The moderate positivity was seen in neoplastic cells outside the nodules; the strong positivity was seen only in the nodules. The different positivity was not linked to the different morphology (round and spindle shape), but it was related to their localization. The Mo showed a moderate positivity. The lymphocytes were negative.

The observations described were done in all cases of thymomas studied. All the above mentioned data are summarized in Table 1.

\section{Discussion}

In the present study the thymic microenvironment was studied from the viewpoint of enzyme histochemistry in normal and pathological conditions (thymomas). The variability of the enzymatic activity was observed in all the thymic cells both in normal and pathological conditions indicating that, in terms of enzymatic activities, the EPCs are a very heterogenous population. In the normal thymus the EPCs show a very high AcP positivity in the cortex and a very weak positivity in the medulla. In the well differentiated thymomas and in the thymomas with spindle epithelial cells, the EPCs show a very weak AcP positivity while in the undifferentiated thymomas they present a strong $\mathrm{AcP}$ reaction. In the well differentiated thymomas and in the undifferentiated thymomas, the EPCs show a very weak or moderate ALP reaction while in the thymomas with spindle epithelial cells they have strong ALP positivity. Ruco et al. [16], showing the distribution and the immunophenotype of Mo and IDCs in the thymomas, have demonstrated that the thymomas had a Mo content that was similar to that of organoid tumours. They have suggested that most thymomas lack the structural organization of the normal thymus; this change has been attributed either to a particular or altered differentiation of the epithelial cells $[12,19]$ or to regression towards a foetal phenotype [8]. Our findings, presenting a different AcP and ALP activity in these types of thymomas, are in agreement with the data and suggestions of the above authors.

Timperley et al. [18] showed that the human thymomas with spindle epithelial cells were negative to ALP. The presence of ALP was of great interest in view of the usual association of this enzyme with cells transporting substance across membranes. On the basis of these data it was and is still likely that the thymus is a secretory organ. On the contrary ALP was totally absent from the thymic tumour cells. This has been explained by the above mentioned authors who assert that if the symptoms of myasthenia gravis are dependent on a secretory product of the tumour cells, this enzyme is not necessary for its secretion. Our data show clearly that ALP activity is present in the thymomas with spindle epithelial cells. This discrepancy could be explained if one accepts that the EPCs may have different histochemical features even if the thymoma has the same histological appearance. The finding is consistent with the fact that the neoplastic cells are a heterogenous population involved in a variety of functional activities. However, these data led us to doubt that a specific type of tumour metabolism really exists.

As regards the LDH and SDH enzymes, Murray [14] has reported that the cancer cells produce lactic acid from glucose in the presence of oxygen. Most tumours showed near-normal levels of respiration, but very high rates of anaerobic and aerobic glycolysis. Our results confirm these findings since we observed a very high LDH and SDH positivity corresponding to the production of energy by the oxidative and non-oxidative metabolism. We suggest that the high rate of aerobic glycolysis exhibited by tumours may be ascribed to the damage of their respiratory-chain energy-coupling mechanism due to neoplastic transformation. The increase in aerobic 
glycolysis could represent a compensatory mechanism of the tumour cells to produce energy (ATP) which is unable to be supplied in amounts sufficient because of the possible damage.

Another interesting finding of this study concerns the accessory cells. Ruco et al. [16] reported that the macrophages were constantly present in the thymomas and that their number and distribution were independent of the histological type of the thymomas. The thymomaassociated macrophages represented the counterpart of the macrophages present in the cortex of a normal thymus and they were necessary to the formation of the clusters of neoplastic cells. These authors showed a slight increase in the number of macrophages per unit area in different types of thymomas. The cells were uniformly distributed throughout the tumour. Our results demonstrate that the macrophage distribution was similar to that reported by Ruco et al. [16], but in the "well differentiated thymomas" we showed that the macrophages were localized in a high number only around the neoplastic nodules that were proliferating (as demonstrated by a very high DHFR positivity). They were absent within the neoplastic nodules. This finding confirms the fact that the macrophages are important for the defence of the organism against the formation of new neoplastic nodules. Moreover, our data suggest that the Mo are a necessary prerequisite for thymocyte survival and may help to explain why the thymomas were rich in these cells.

\section{References}

1. Beller, D. I. and Unanue, E. R.: Ia antigen and antigen-presenting function of thymic macrophages. J. Immunol. 124; 1433$1441,1980$.

2. Bancroft, J. D. and Hand, N. M.: Demonstration of dehydrogenases. In "Enzyme Histochemistry", ed. by Royal Microscopical Society, Oxford University Press, Oxford, 1987, pp. 53-56.

3. Boyd, R. L., Tucek, C. L., Godfrey, D. I., Izon, D. J., Wilson, T. J., Davidson, N. J., Bean, A. G. D., Ladyman, H. M., Ritter, M. A. and Hugo, P.: The thymic microenvironment. Immunology Today 14; 445-456, 1993.

4. Free, M. J.: Carbohydrate metabolism in the testis. In "The Testis", ed. by A. D. Johnson, W. R. Gomes and N. L. Van Denmark, vol. 2, Academic Press, New York, 1970, pp. 125292.

5. Gerzeli, G. and De Picies Polver, P.: Studio citochimico della tetraidrofolato deidrogenasi nel sangue di pollo nel corso dell'in- dagine sperimentale con Plasmodium gallinaceum. Riv. Parassitol. 30; 19-25, 1969.

6. Gomori, F.: Alkaline phosphatase of cell nuclei. J. Lab. Clin. Med. 37; 526-530, 1951.

7. Grogg, E. and Pearse, A. G. E.: A critical study of the histochemical techniques for acid phosphatase with a description of an azo-dye method. J. Path. Bact. 64; 627-631, 1952.

8. Haynes, H.: The human thymic microenvironment. In "Advances in Immunology”, ed. by F. J. Dixon, Vol. 36, New York, Academic Press 1984, pp. 87-92.

9. Ho, F. C.S., Fu, K. H., Lam, S. Y., Chin, S. W., Chan, A. C. L. and Muller-Hermelink, H. K.: Evaluation of a histogenetic classification for thymic epithelial tumours. Histopathol. 25; 21-29, 1994.

10. Kodoma, T., Watanabe, S., Sato, Y., Shimostao, Y. and Miyazawa, N.: An immunohistochemical study of thymic epithelial tumours. I. Epithelial component. Am. J. Surg. Pathol. 10; 26-33, 1986.

11. Mosier, D. E. and Pierce, C. W.: Functional maturation of thymic lymphocyte population in vitro. J. Exp. Med. 136; 1484-1500, 1972.

12. Mokhtar, R. N., Hsu, S., Lad, R. P., Haynes, B. and Jaffe, E.: Thymoma: lymphoid and epithelial component mirror the phenotype of normal thymus. Human Pathol. 15(4); 378-384, 1984.

13. Muller-Hermelink, H. K., Palestro, M. M., Palestro, G., Schumacher, U. and Kitcher, T.: Immunohistological evidence of cortical and medullary differentiation in thymoma. Virchows Arch. [A] 408; 143-161, 1985.

14. Murray, R. K.: Biochemical properties of cancer cells. In "The Basic Science of Oncology", ed. by I. Tannack, R. Hill, Ontario Cancer Institute and Univ. Toronto. Pergamon Press, Toronto, 1988, pp. 176-191.

15. Owen, J. T.: The origin and development of lymphocyte population. Ontogeny of Acquired Immunity. Ciba Found. Symp. North Holland, Elsevier, 1972, p. 35.

16. Ruco, L. P., Rosati, S., Monardo, F., Pescarmona, E., Rendina E. A. and Baroni, C. D.: Macrophages and interdigitating reticulum cells in normal thymus and in thymoma: an immunohistochemical study. Histopathol. 30; 59-650, 1980.

17. Ruco, L. P., Pisacane, A., Pomponi, D., Stoppacciaro, A., Pescarmona, E., Rendina, E. A., Santoni, A., Baraschi, D., Tagliabue, A., Uccini, S. and Baroni, C. D.: Macrophages and interdigitating reticulum cells in normal human thymus and thymomas: immunoreactivity for interleukin 1, interleukin 1 beta and tumour necrosis factor alpha. Histopathol. 17; 291299, 1990.

18. Timperley, W., Path, M. R.C. and Ahmed, A.: Histochemistry of the thymus and a thymoma. Arch. Path. 89; 405-410, 1970.

19. Wilcox, N., Schluep, M., Ritter, M. A., Schuurman, H. J., Newsam, D. J. and Chrisensson, B.: Myasthenic and nonmyasthenic thymoma. An expansion of a mirror cortical epithelial cells subset? Am. J. Pathol. 127; 447-460, 1987. 